\title{
An IoT-aware Architecture to improve Safety in Sports Environments
}

\author{
Luca Catarinucci, Danilo De Donno, Luca Mainetti, Luigi Patrono, Maria Laura Stefanizzi, and \\ Luciano Tarricone
}

\begin{abstract}
The introduction of Internet of Things enabling technologies into the sport and recreational activities domain provide an interesting research challenge. Their adoption could significantly improve the sport experience and also the safety level of team sports. Despite this, only few attempts have been done to demonstrate the benefits provided by use of IoT technologies in sport environments. To fill this gap, this paper propose an IoTaware Sport System based on the jointly use of different innovative technologies and standards. By exploiting the potentialities offered by an ultra-low-power Hybrid Sensing Network (HSN), composed of 6LoWPAN nodes integrating UHF RFID functionalities, the system is able to collect, in real time, both environmental parameters and players' physiological data. Sensed data are then delivered to a Cloud platform where a monitoring application makes them easily accessible via REST Web Services. A simple proof of concept has demonstrated the appropriateness of the proposed solution.
\end{abstract}

Index Terms-Internet of Things; Sport; Cloud; Wireless Sensor Network; mobile.

\section{INTRODUCTION}

$\mathrm{S}$ MART environments are expected to become the main actors of the Future Internet, which will be no longer seen as a mean to connect people to services but to access the resources made available by small smart objects. This vision of the Internet fits into the broader concept of the Internet of Things (IoT), according to which everyday objects, such as domestic appliances, actuators, and embedded systems of any kind, will become proactive actors of the global Internet, with the capability of generating and consuming information. By exploiting the new possibilities offered by the IoT enabling technologies, innovative smart applications could be developed in many heterogeneous scenarios, such as home and industrial automation, healthcare and wellness, smart grids, automotive, and many others [1], [2]. Among these, sports and recreational activities provide interesting research areas that

Manuscript received January 13, 2017; revised May 12, 2017. Date of publication: June 1, 2017.

Authors are with the Innovation Engineering Department, University of Salento, Via per Monteroni, 73100, Lecce, Italy.

Luigi Patrono is the corresponding author (e-mail: luigi.patrono@unisalento.it).

Digital Object Identifier (DOI): 10.24138/jcomss.v13i2.372 include several of the critical challenges for next generation of services in a variety of scenarios.

Football is one of the most popular sports in the world and, therefore, it could represent an interesting starting point for the introduction of IoT technologies into sport environments. FIFA, the organization responsible for developing and improving the quality of football, periodically introduces innovations in the football field, such as Goal Line Technology (GLT), in order to improve the game experience [3], [4]. FIFA also aims to make football a safe sport. Concussion, hypoglycemia, heartbeat irregularities and shortness of breath are some of the injuries to which players are subjected during a football match [5]. The adoption of emerging IoT technologies in the football field could help coaches and doctors to identify possible emergency situations and promptly help a player in trouble.

Among all the IoT-enabling technologies suitable for the development of innovative systems, Ultra High Frequency (UHF) Radio Frequency Identification (RFID), and Wireless Sensor Network (WSN) represent two of the most promising solutions. UHF RFID is a short-range radio technology mainly used for object identification and tracking within quite small areas [6], while IEEE802.15.4-based WSNs are particularly suited for environmental monitoring and localization purposes even in harsh environments [7]-[10]. They represent two complementary technologies, whose physical integration might give new perspectives to a broad range of innovative applications [11], [12]. By merging RFIDs with WSNs, indeed, important information such as object identity and location, but also environmental conditions, could be retrieved. Moreover, RFID systems integrated with WSNs could benefit from the multi-hop wireless communication, thereby overcoming the main drawback of the RFID technology (i.e. the single-hop communication with the reader).

To maximize the benefits provided by a such an integration, the adoption of IoT protocols is advocated. In such way, RFIDWSN integrated devices could become remotely accessible though the Internet, thus favouring the development of novel smart environments. One of the most used communication protocol in the IoT is Constrained Application Protocol (CoAP) [13]. CoAP allows sensor nodes to run embedded Web services, through which their resources can be easily manipulated. Furthermore, the development of REST- style IoT systems 
enables seamless interoperability with Cloud services, thus guaranteeing the implementation of easily scalable solutions.

The described concepts have been already successfully applied for the development of innovative solutions, especially in the healthcare domain. An interesting project, which aims to

patients' monitoring and tracking, is WSN4QoL [14]. In [15], a WSN architecture and smart mobile communication techniques are combined to monitor the health condition of patients and provide several effective healthcare services. An interesting attempt that aims to combine and integrate heterogeneous technologies is reported in our prior work [12]. More in depth, it describes a smart system based on UHF RFID and WSN solutions for the automatic monitoring and tracking of patients within hospitals. However, such system works properly only within a closed environment, such as a hospital, since it does not represent a scalable and flexible solutions.

However, there is a lack of literature on applying IoTenabling technologies in the area of sport. In [16], authors discuss the main research challenges related to the adoption of IoT architectures in the sport domain. In [17], an IoT-based architecture for the sport of football, called IoT Football, is proposed. The system embeds sensing devices, telecommunication technologies (e.g. ZigBee) and cloud computing in the football sport in order monitor the health of footballers and reduce the occurrence of adverse health conditions. The described solution, however, is only at a first stage of development, since it was not still implemented and tested using real devices.

To fill this gap, in this work, a novel IoT-aware Sport System, able to guarantee innovative services for the automatic monitoring of players during a match, is described and validated. It is based in the jointly use of different, yet complementary, technologies and standards (e.g., RFID, WSN, mobile, 6LoWPAN, and CoAP). The designed solution has been customized and prototyped for the game of football, due to its great popularity, however, its design is generic enough to be easily applied to other team sports (i.e., volleyball, basketball, rugby, tennis). More in depth, the system is able to collect both environmental conditions and players' physiological parameters via an ultra-low-power Hybrid Sensing Network (HSN). This last one describes a typical 6LoWPAN network embedding also two new kinds of WSN nodes integrating UHF RFID Class-1 Generation-2 (Gen2 hereafter) functionalities. The former integrates an RFID Gen2 reader while the latter integrates an augmented RFID Gen2 tag. In such way, physiological parameters of player can be easily retrieved by RFID Gen 2 readers deployed near the benches located in the football field. The retrieved data is then delivered to a Cloud platform, where a customized monitoring application makes them easily accessible via REST Web services. During the normal situation, therefore, no WSN-based transmission is performed, thus reducing the node power consumption and limiting the impact on the network capacity. The designed system is also able to timely and reliably manage emergency situations. In this case, indeed, the WSN-based transmission is activated so as to promptly inform coaches and doctors in the field. Doctors, equipped with a smartphone connected to a portable UHF RFID reader, can use a customized mobile application to interact with players' sensor nodes during their interventions. Finally, the system allows also to retrieve and store important data about the environmental conditions, which could be used to provide alternative services to citizens or researchers, within the realization of a Smart City infrastructure.

It is worth to notice that this work represents an extended version of the solution already presented in [18]. Respect to our previous paper, in this work the proposed solution has been analyzed in more detail and deeper validated, through test bed on a real football field.

The rest of the paper is organized as follows. Section II provides the technological background. Section III describes the overall architecture design, while the RFID-WSN integration approach is summarized in Section IV. A more detailed description of the system is presented in Section $\mathrm{V}$, while a prototype implementation of the proposed system is described and validated in Section VI. Concluding remarks are drawn in Section VII.

\section{TECHNOLOGICAL BACKGROUND}

\section{A. RFID and WSN}

RFID is a low-cost and low-power technology mainly consisting of passive devices, called tags, which are able to transmit data when powered by the electromagnetic field generated by an interrogator, called reader. Such devices are usually classified according to the frequency band used (e.g., LF, HF, UHF) and the type of coupling (i.e., magnetic and electromagnetic) between tag and reader antennas. Since passive RFID tags do not need a source of energy to operate, their lifetime can be measured in decades, thus making the RFID technology well suited in a variety of application scenarios. The recent availability of UHF RFID tags with increased capabilities, e.g. sensing and computation, represents a further added value. Nevertheless, the main drawback of RFID tags stems from the fact that they can operate solely under the reader coverage region. Such an aspect limits the use of UHF RFID technology to object identification and monitoring within small areas [6].

WSNs consist of a large number of low-cost, low-power embedded devices, called sensor nodes, which are able to selfconfigure and self-organize. These characteristics make them suitable to be deployed even in harsh environments in order to detect important parameters (e.g. temperature, light, humidity, etc.) without human intervention. The collected data are then delivered, in a multi-hop fashion, to a central point (sink) for proper utilization. This simple, yet fundamental functionality, has enabled the widespread adoption of WSN technology in many heterogeneous scenarios, such as building automation, surveillance, military operations, healthcare, logistics, etc. However, one of the main issues to be addressed in the development of complex applications is the power consumption. WSN motes, indeed, are usually battery-powered computing platforms integrating analog/digital sensors and an IEEE 802.15.4 radio enabling up to $100-\mathrm{m}$ outdoor communication range (single hop). They are typically deployed in large areas where changing or replacing batteries could be impractical or completely unfeasible. For this reason, the use of 
effective solutions to increase the network lifetime is fundamental in real applications [7][8].

\section{B. Constrained Application Protocol (CoAP)}

Well-known and widely used Internet protocols are often unsuited for IoT devices, which are usually constrained, especially in terms of computational power, memory, and transmission bandwidth. For this reason, in the last years, many researchers focused their activities on designing protocols for the IoT. One of the main outcomes of this research effort is Constrained Application Protocol (CoAP)[12], a specialized Web transfer protocol defined by the IETF Constrained RESTful Environments working group to allow the implementation of REST mechanisms on constrained devices.

The main idea of this protocol is to provide a lightweight access to physical resources in order to meet the limited capabilities of embedded devices, such as WSN nodes. CoAP provides a request/response model interaction between two end-points and it includes key concepts of the Web as URI and media types. CoAP, like HTTP provides the following four methods for resources' manipulation: (i) GET, to retrieve a representation of the resource identified by the request URI; (ii) POST, to request that the representation enclosed in the request is processed; (iii) PUT, to request that the resource identified by the URI is updated or created with the transmitted representation; (iv) DELETE, to request that the resource identified by the specified URI is deleted.

In addition to the HTTP features, it offers a built-in mechanism for the resources discovery, it supports the IP multicast, and it natively provides a server-push model and an asynchronous exchange of messages. It also has a small-size header in order to be used on low-power networks like 6LoWPAN over IEEE 802.15.4. It can also run on top of proprietary networks that are connected to IPv6 Internet. Moreover, it bases the communication on the UDP for reducing the communication costs. CoAP also provides a resource observation mechanism, which allows a client to receive notifications upon every change in the state of resources it has previously subscribed to.

\section{System ARCHITECTURE DESIGN}

The designed system has been put into effect according to the architecture illustrated in Fig. 1. As shown, it is composed of four main parts: (1) the Hybrid Sensing Network (HSN), (2) the IoT Smart Gateway, (3) the cloud platform, and (4) the user interfaces.

The HSN consists of an integrated RFID-WSN 6LoWPAN network composed of four types of nodes: (i) 6LowPAN Border Router (6LBR), (ii) 6LowPAN Router (6LR), (iii) 6LowPAN Router Reader (6LRR), and (iv) 6LowPAN Host Tag (HT). According to the 6LoWPAN standard, the 6LBR is in charge of connecting the network to the Internet by translating 6LowPAN packets into IPv6 packets and vice-versa, a 6LR describes a node able to provide forwarding and routing capabilities, while the 6LRR is defined as a 6LR interfaced with an RFID reader. Finally, an HT node identifies a typical 6LowPAN Host (i.e. a node without routing and forwarding capabilities) interfaced with an RFID Gen2 tag. The designed system assumes that 6LR, equipped with light, humidity and temperature sensors, are deployed along the perimeter of playing field in order to detect environmental conditions, while 6LRR nodes are placed on poles located near benches reserved for players. Indeed, the main function of 6LRR nodes is to identify and monitor players labeled with RFID Gen 2 tags. More in detail, players wear a HT node capable to detect important physiological parameters, such as body temperature and heartbeat. During the match, sensed data are periodically logged on the memory of the RFID Gen2 tag. In this way, 6LRR nodes can retrieve such information when players approach the benches (as example, at the end of a playing period) and delivered to the IoT Smart Gateway.

In the envisioned architecture, IoT Smart Gateway is a more powerful device placed near the exit from the playing field. It is connected, on the one hand, directly with the HSN and, on the other hand, with the Internet through a $3 \mathrm{G}$-communication interface. The gateway, therefore, plays the role of $6 \mathrm{LBR}$, enabling the communication between HSN nodes and remote users. Moreover, the gateway enables the RESTful communication with the cloud platform.

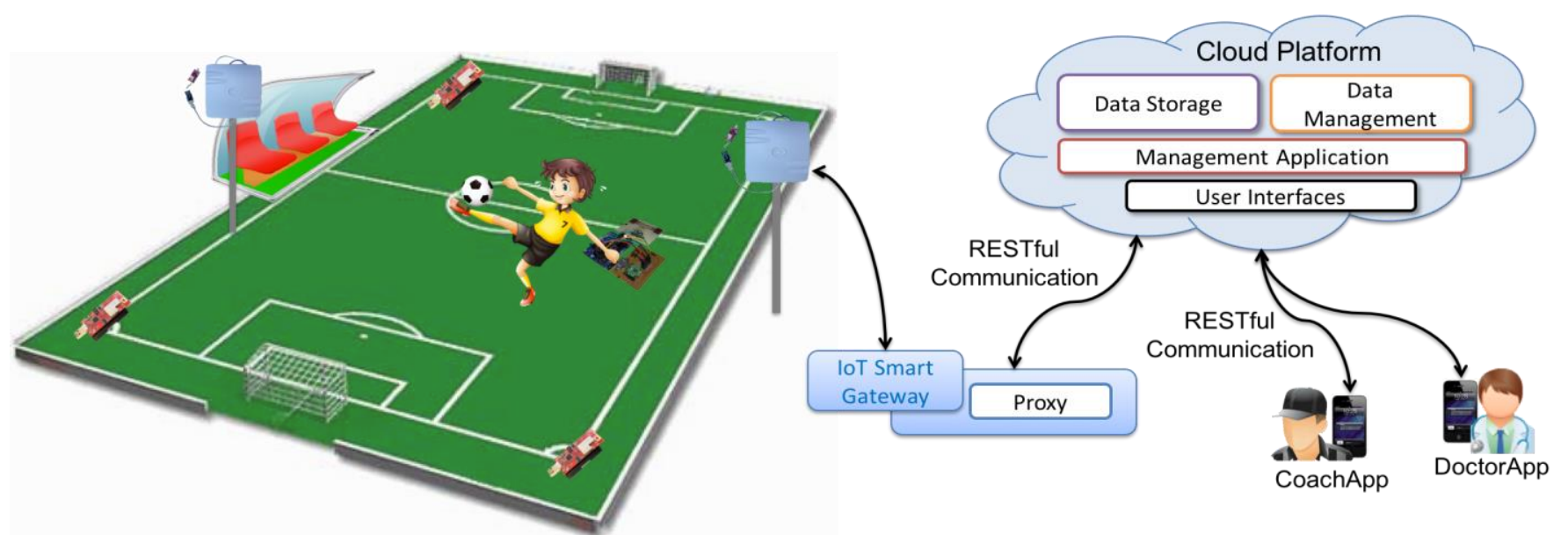

Fig. 1. Overall system architecture 
This last one is equipped with three different modules: (i) a Data Storage Module, in charge of storing the received data; (ii) a Data Management Module, responsible to remotely control and manage sensors deployed in the field; and (iii) a Management Application (MA), able to execute the business logic.

In particular, the MA module plays a key role within the developed architecture. It is responsible to timely manage emergency situations. As previously introduced, in case of critical events, such as player's heartbeat irregularities, the HT node activates its long-range IEEE 802.15.4 radio transceiver to send a notification to the MA. This last module is able to analyze heterogeneous information (i.e., the environmental conditions as well as the player's physical condition) in order to detect potentially dangerous situations. In such a case, the MA exploits Push Notifications (PN) to inform the coach and the doctor in the field that the player needs to be immediately taken off the field and provided with medical support. This strategy allows the HT nodes to always use the RFID Gen2 radio interface for routine operations, e.g. data logging and identification, while keeping the IEEE 802.15.4 radio off for most of the time, thus maximizing battery lifetime.

To make the collected data easily accessible by users, two different mobile applications have been developed. In the designed architecture, doctors are equipped with a smartphone connected to a portable RFID Gen2 reader and running a customized application, named DoctorApp. Through this App, during the playing breaks, doctors can interact directly with the HT node worn by a player and check her/his physiological parameters by reading the most recent information stored into the memory of the RFID Gen2 tag or historical information stored into the Cloud. The DoctorApp allows doctors also to update the tag's memory content with information to remind for the time of the football match (e.g. the time of the last check), and receive notifications in case of emergency. A different mobile application, called CoachApp, allows football coach to check data about the environmental conditions and to receive notifications when an emergency is detected. Since the system collects sensitive and confidential data, the platform must ensure an adequate level of security to data access and management. For this reason, users need to be authenticated before they can access the platform.

\section{RFID AND WSN INTEGRATION}

In Fig. 2 the proposed RFID-WSN integration approach is sketched: a conventional UHF RFID tag featuring the $\mathrm{I}^{2} \mathrm{C}$ interface is physically connected to the microcontroller unit (MCU) of a WSN node. In this way, information exchanged between the tag and the RFID reader via the EPCglobal Class1 Generation-2 (Gen2) air interface are directly available also on the $\mathrm{I}^{2} \mathrm{C}$ interface and, therefore, accessible by the MCU. Potentially, the proposed RFID-WSN integration enables EPCglobal standardized data to be spread, in a multi-hop fashion, throughout an IEEE 802.15.4 network.

In particular, the RFID Section of Fig. 2 has been realized through the prototype shown in Fig. 3.

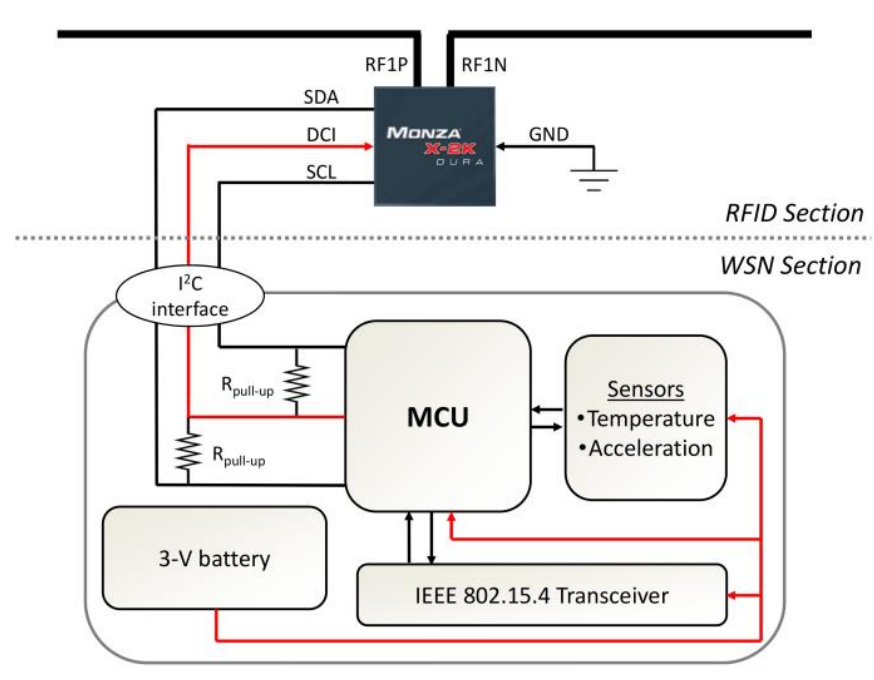

Fig. 2. Proposed integration approach between RFID and WSN. Red connections show how the on-board power is routed to the functional blocks.

The main elements are an antenna, a chip, and a battery older. The battery older could be used to enable a stand-alone mode of operation as a battery-assisted passive (BAP) RFID tag, even though it is not exploited in the proposed RFID-WSN integration since the required power is fed directly from the 3$\mathrm{V}$ battery of the WSN node.

The used chip is an Impinj Monza X-2K RFID chip, which is a new-generation UHF RFID Gen2 integrated circuit (IC) with 2176 bits of non-volatile memory (NVM) and an $\mathrm{I}^{2} \mathrm{C}$ interface. As an $\mathrm{I}^{2} \mathrm{C}$ device, Monza X-2K operates as a standard EEPROM whose contents can also be accessed via the Gen2 air interface. In the fabricated prototype, the small female header, which exposes the $\mathrm{I}^{2} \mathrm{C}$ bus, is used to interface the RFID chip with the WSN Section thus physically realizing the RFID-WSN integration sketched in Fig. 2.

As the input impedance of the chip at $866.5 \mathrm{MHz}$, i.e. the center frequency of the European UHF RFID band, is $\mathrm{Z}_{\text {chip }}=\mathrm{R}_{\text {chip }}+\mathrm{j} \mathrm{X}_{\text {chip }}=20.83-\mathrm{j} 181.39 \Omega$, the designed antenna has been optimized to guarantee the conjugate matching with such impedance. More in detail, the designed dipole-like UHF RFID antenna takes cue from the commercial ALN-9660 RFID tag inlay which uses meander lines to achieve a very compact form factor (i.e., $7.5 \times 1.7 \mathrm{~cm}^{2}$ ). Detailed simulations taking into account the effect of the DC metal traces have been carried out in order to maximize the performance and optimize the design of the final prototype. Three main elements define the antenna structure: (i) the central loop, which primarily impacts on the tuning of the real part of the input impedance and prevents potential high-voltage discharge, (ii) the meander lines to reduce the antenna size, and, finally, (iii) the capacitive loads at the antenna tips, which facilitate the impedance matching. Simulations were performed by the CST MW Studio setting the input impedance of the antenna port equal to $\mathrm{R}_{\text {chip }}$ and adding a capacitive lumped element with reactance $\mathrm{X}_{\text {chip. }}$ In this way, the problem is led back to the design of classic antennas with real impedance. 


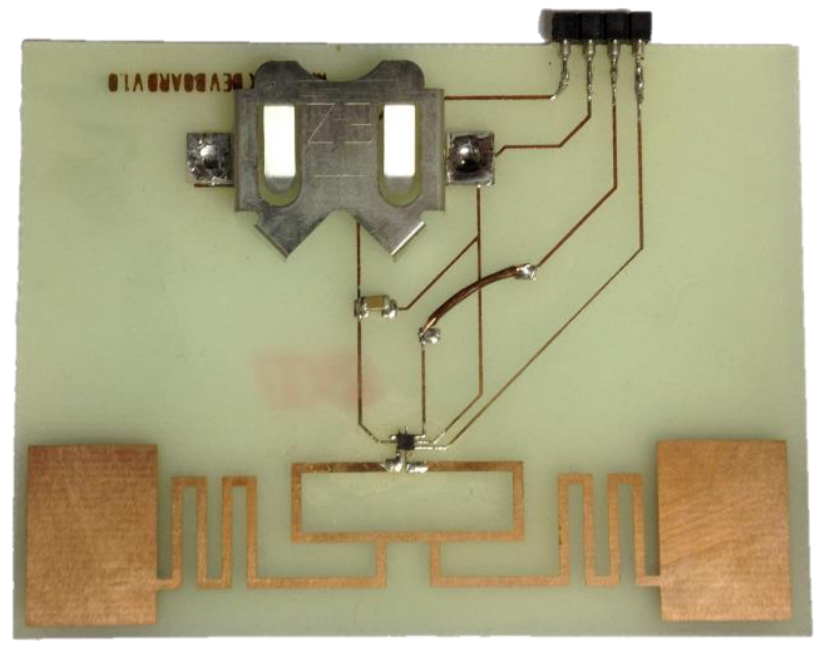

Fig. 3. Prototype of the developed RFID tag equipped with a small female header exposing the $I^{2} \mathrm{C}$ interface (dimensions: $7.5 \times 5 \times 1.6 \mathrm{~cm}^{3}$ ).

Finally, the antenna design has been optimized by setting the minimization of the reflection coefficient at the desired frequency as the fitness function and by adopting a gradientbased interpolated quasi-newton optimizer. A detailed comparison between simulation and measurement results for the proposed RFID tag antenna is reported in [19].

The RFID board is interfaced via the $\mathrm{I}^{2} \mathrm{C}$ bus to the MB851 board developed by ST Microelectronics. This board (representing the WSN Section in Fig. 2) is equipped with a 32bit ARM Cortex-M3 MCU operating up to $24 \mathrm{MHz}$ and embedding 16-KB RAM and 256-KB eFlash ROM. It integrates also a $2.4 \mathrm{GHz}$ wireless transceiver compliant with the IEEE 802.15.4 standard. The featured MCU is optimized to provide high performance at very low power consumption. The WSN board is also equipped with an external antenna connector and 24 highly configurable GPIOs with Schmitt trigger inputs. Among the exposed GPIOs, the SDA and SCL lines, the ground pin (GND), and an output pin (DCI) have been used to implement the $\mathrm{I}^{2} \mathrm{C}$ communication with the RFID Section and provide power to the RFID chip.

The performance of the proposed hybrid RFID-WSN device has been extensively evaluated through experiments. Among the several evaluations we performed, the power consumption is of particular interest to be reported, being the available energy provided by the on-board battery a critical constraint when designing real-world WSN applications. We used an Agilent DSO-X-2012A digital oscilloscope to track the voltage drop across a $1-\mathrm{k} \Omega$ precision shunt resistor connected in series with the RFID-WSN node under 3-V supply voltage. Then we calculated the absorbed DC current under different operating conditions by the Ohm's law. Our analysis showed that the current absorbed by the node varies between $1.5 \mu \mathrm{A}$ and $35 \mathrm{~mA}$ depending if the operating mode is set to idle/fully-passive or active, respectively.

In addition to the power consumption, the achievable RFID Gen2 communication range is another fundamental aspect to evaluate. The maximum communication range was measured experimentally by using a Gen2 RFID reader set with 33-dBm transmit power and configured to perform read/write operation from/to the user memory of the Monza X-8K chip at different distances. The experiments were conducted in a large lecture room with reader and RFID-WSN node placed in the line of sight (LOS) $1.5 \mathrm{~m}$ above the floor, both oriented in the maximum-gain direction. The distance between the two devices was increased in step of $0.5 \mathrm{~m}$ and, for each measurement point, the reader was instructed to perform 100 attempts to access the Monza X-8K user memory. Our experiments revealed that the memory read/write success ratio remains above $80 \%$ up to 13 $\mathrm{m}$ and $22 \mathrm{~m}$ when the RFID Gen2 section is configured in fullypassive and BAP mode, respectively.

\section{SySTEM DETAILS}

In the following, implementation details on the designed software system are described.

\section{A. Hybrid Sensing Network}

As already introduced, key elements of the designed HSN are the HT nodes and 6LRR devices. The former has been described in the previous section, while the latter consists of a commercial off-the-shelf (COTS) RFID Gen2 Reader interfaced with the XM1000 mote from Advanticsys [19] via the universal asynchronous receiver/transmitter (UART) communication bus. Specifically, the Sensor ID Discovery Gate UHF [20] reader has been used.

As summarized in Table 1, three different kinds of resources can be identified in the proposed architecture: (i) health sensors, (ii) ambient sensors, and (iii) RFID-related resources. 6LR nodes scattered in the football field can monitor only environmental parameters and, therefore, expose just CoAP ambient sensor resources (e.g. coap://[aaaa::1]/ambient/light and coap://[aaaa::1]/ambient/temperature). In addition to such kind of resources, 6LRR nodes can expose an RFID resource (coap:// [aaaa: :3] /RFID/reader) which represents an aggregated information of tags read within the 6LRR RFID range. Finally, HT nodes also expose an RFID resource (coap://[aaaa::3]/RFID/tag, which identifies the memory content of the integrated Gen $2 \mathrm{tag}$ ) in addition to both ambient and health (e.g. coap: // [aaaa: :3] /health/heartbeat, which provides sensor readings from the integrated 3-axis accelerometer) sensor resources. In this way, each resource can be individually accessed from anywhere in the Internet by using CoAP methods.

For the sake of simplifying the development of the proposed solution, we drawn on the implementation presented in [21] where Erbium (Er), a low-power REST engine for Contiki, has

TABLE 1.

TYPOLOGIES OF RESOURCES AND RELATED COAP PATHS

\begin{tabular}{|c|c|c|}
\hline Node & Resource & Examples of resource path \\
\hline 6LR & $\begin{array}{l}\text { Env. } \\
\text { Sensor }\end{array}$ & $\begin{array}{l}\text { coap://[aaaa: :1]/ambient/light } \\
\text { coap://[aaaa::1]/ambient/temp }\end{array}$ \\
\hline 6LRR & $\begin{array}{l}\text { Env. } \\
\text { Sensor } \\
\text { RFID } \\
\text { Reader }\end{array}$ & $\begin{array}{l}\text { coap://[aaaa: :2]/ambient/light } \\
\text { coap://[aaaa: :2]/ambient/temp } \\
\text { coap://[aaaa: :2]/RFID/reader }\end{array}$ \\
\hline HT & $\begin{array}{l}\text { Env. } \\
\text { Sensor } \\
\text { Health } \\
\text { Sensor } \\
\text { RFID Tag }\end{array}$ & $\begin{array}{l}\text { coap://[aaaa: :3]/ambient/light } \\
\text { coap://[aaaa: : 3]/health/heartbeat } \\
\text { coap://[aaaa: :3]/RFID/tag }\end{array}$ \\
\hline
\end{tabular}


been extended to support conditional observations through a Conditional Observation Module. Such implementation has been adapted to our hardware. Specifically, in the proposed solution, the HT node embeds sensors able to monitor not only environmental conditions, but also vital signs, e.g. heartbeat, which should maintain predefined values in players with good health conditions. However, if their values fit outside a specified range, it might indicate the player needs attention. The use of conditional observation methods allows client applications to be notified only when critical thresholds are violated.

\section{B. Smart Gateway}

The Smart Gateway is another important element of the designed architecture. It works as a bridge between the HSN and the Cloud platform. It has been realized by connecting a Rasperry Pi 2 Model B board [22], equipped with the Raspian operating system, to the 6LowPAN Border Router. The gateway has been also equipped with a $3 \mathrm{G}$ module, to guarantee data communication. Raspberry $\mathrm{Pi}$ is a credit card-sized computer powered by the Broadcom BCM2836 system-on-achip (SoC). This SoC includes a quad-core ARM Cortex-A7 CPU, clocked at $900 \mathrm{MHz}$. It is equipped with $1 \mathrm{~GB}$ of RAM and powered by a $5 \mathrm{~V}$ micro USB AC charger.

In our implementation, the Smart Gateway embeds a proxy subsystem, which enables transparent communication with CoAP devices. It has the burden of translating HTTP requests coming from the Cloud platform into CoAP messages and viceversa. It has been developed by using the Spring Framework and deployed on the Jetty application server installed on the Smart Gateway. The proxy logic has been extended by implementing a caching service, thus supporting multiple requests to the same resource and limiting the amount of traffic injected into the IoT peripheral network. This feature is particularly important for constrained nodes, which are not able to simultaneously manage requests from multiple clients.

\section{Cloud Services}

Storing, organizing, and retrieving information related to the players' performance during a match and environmental conditions are expensive processes from both the computational and memory point of view. For this reason, the cloud seems to represent the solution that best suits this kind of needs, as its storing and computing capabilities allow to process data more efficiently.

In the proposed system, a cloud platform has been used to store and manage the information retrieved by the HSN and all the data related to players' health conditions. We deployed the proposed solution on Amazon Elastic Compute Cloud (EC2). Specifically, as previously introduced, the cloud platform has been equipped with the following modules: (i) Data Storage Module, (ii) Data Management Module, and (iii) Management Application (MA). In particular, the last one represents the functional core of the proposed architecture. More in depth, the MA registers itself as an observer to the CoAP resources exposed by HT nodes and to the RFID reader related resources exposed by 6LRR nodes deployed near the benches. In such a way, when a football player enters within the coverage region of a 6LRR node, all the information stored into the memory of the RFID Gen2 chip can be read and delivered to the MA for a further analysis. At the same time, the use of conditional observation methods allows the MA to be notified only when the value of player's physiological parameters fit outside a specified range, thus substantially reducing the number of notification messages in the network. The MA is also able to send Push Notifications to the mobile devices of coaches and doctors. To this purpose, we resorted to the Amazon Simple Notification Service (SNS), since it can seamlessly scale and add an abstraction level allowing programmers to use the same APIs for sending notifications on different platforms (e.g. iOS and Android).

\section{Mobile Applications}

Users can interact with the system through user interfaces, accessible via Web browser by both desktop clients and mobile devices. Such interfaces implement RESTful services, thus enabling the communication with the Cloud platform. They offer two main functionalities depending on two possible client profiles:

Coach Interface. This interface allows coaches to visualize current and historical information from environmental sensors, and data about players' performance as well. Coaches can interact remotely with the system by using the CoachApp, a customized Android application. As previously described, the App also allows coaches to receive notifications in case of emergency situations.

Doctor Interface. This interface allows doctors to visualize information about players' health status and to access to the health sensor data of each patient wearing an HT node. Doctors can interact remotely with the system by using the DoctorApp, a customized Android application. As previously described, the DoctorApp also allows doctors equipped with an RFID-enabled smartphone to directly retrieve and manage player's healthy data stored into the tag's memory during the football match breaks. Finally, the DoctorApp is able to receive PNs in case of emergency.

\section{PROOF-OF-CONCEPT}

In this section, a prototype implementation of the proposed system is described and validated by means of a simple proof of concept.

Specifically, the ADXL345 digital 3-axis accelerometer connected to the multi-sensor board of the HT node has been exploited to detect possible heart problems and generate an alert. According to the algorithm proposed in [23], the signal is initially passed through a band-pass filter. Next, the algorithm computes the first and second derivative of the signal using two approximate 5-tap filters with integer coefficients. The results are added together and considered in absolute value. A 6-tap moving average is then applied, and the result compared against a threshold to detect any peaks that may indicate physiological issues. It is worth to notice that the aim of this paper is to demonstrate the feasibility of the proposed system. Therefore, the definition of specific, optimum algorithms to detect heartbeat irregularities is outside the scope of this work. 


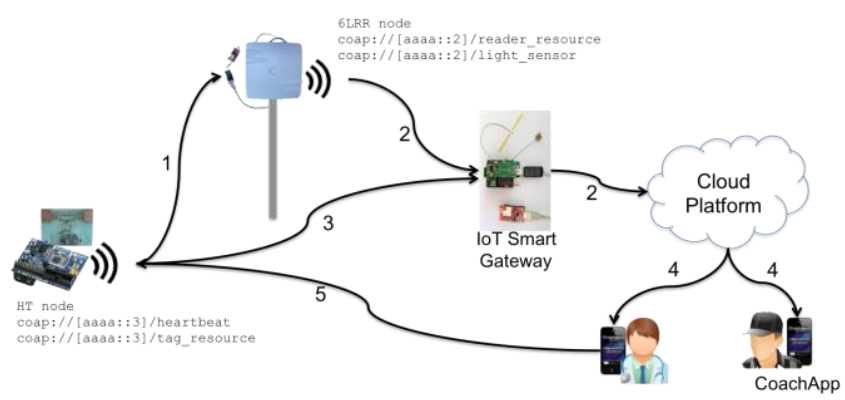

Fig. 4. Use case scenario

The considered validation scenario is depicted in Fig. 4. The two main actors are: (i) the HT node, in charge of monitoring the player's health status, and (ii) the 6LRR node, in charge of reading and delivering to the IoT Smart Gateway data retrieved from the memory of the RFID chip equipping the HT node. The RFID tag embedded into the HT node contains, in addition to player's physiological information, the Electronic Product Code (EPC), used to univocally identify the football player. A Nexus 4 mobile phone running Android 4.4.3 "KitKat" connected to the BlueBerry RFID Gen2 reader from TERTIUM Technology [24] is used as handled reader. The DoctorApp is installed on the Nexus 4 and uses the Wi-Fi or $3 \mathrm{G}$ Internet access. When an irregular-heartbeat event is detected the application receives the PN sent by the MA and notifies the doctor with a sound. The DoctorApp also allows the doctor to retrieve from the cloud further details on the occurred event and historical data about the player's health status.

To demonstrate the efficiency of the proposed system, two different use cases have been considered: (i) players' monitoring and (ii) emergency event handling. In the former, the following operations are performed:

1. the 6LRR identifies the player by means of her/his unique EPC and retrieve her/his current and historical health status by accessing the memory of the RFID chip equipping the HT node;

2. the 6LRR node sends a notification message containing the read data to the MA using a CoAP method. The MA application running on the cloud analyze the received data and store them.

In the latter, the following procedure is used to handle emergency situations:

3 . the HT node detects a heartbeat irregularity and activates its IEEE 802.15.4 radio transceiver to send a notification message to the MA;

4. the MA analyses the received data and send a PN to coaches and doctors in the field.

5. The doctor receives on the DoctorApp the emergency notification (Fig. 5a) and visualizes details about the emergency event by reading data stored in the RFID tag (Fig. 5b).

At the end of the football match the coach uses the CoachApp to visualize the player's performance (Fig. 6).

\section{CONCLUSION}

In this paper, a novel Sport System architecture for automatic monitoring of players has been proposed. Let us observe that this work represents an extended version of a previously presented paper. Respect to this last one, more details about the designed architecture have been presented. Furthermore, the Sport System has been extensively validated on a real football field.

More in depth, according to the IoT vision, a complete network infrastructure relying on a CoAP, 6LoWPAN, and REST paradigms has been implemented so as to allow the
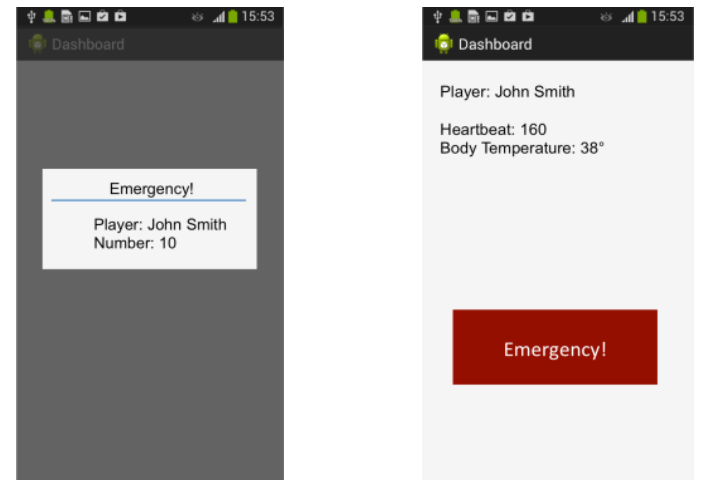

Fig. 5. Screenshots of the DoctorApp: (a) PN on the mobile phone; (b) visualization of details about the emergency situation.

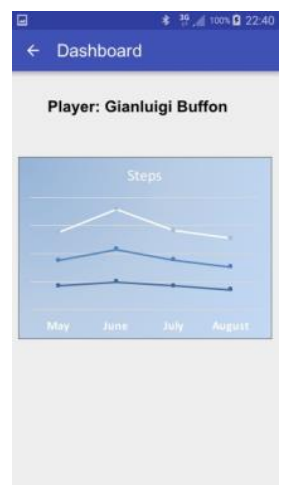

Fig. 6. Screenshot of the CoachApp.

interoperation among UHF RFID Gen2, WSN, and smart mobile technologies. The designed solution has been prototyped for the game of football, due to its great popularity. In particular, an ultra-low-power Hybrid Sensing Network (HSN), able to collect the real-time variation of any critical players' physiological parameter as well as of the environmental conditions, has been implemented. The sensed parameters are delivered to a Cloud platform and made easily accessible via customized REST Web Services. To validate the proposed system, two different use cases have been implemented. The former deals with players' monitoring, the latter with the management of an emergency situation. The achieved results demonstrate the appropriateness of the proposed system.

\section{REFERENCES}

[1] L. Mainetti, L. Patrono, and A. Vilei, "Evolution of wireless sensor networks towards the Internet of Things: A survey," $19^{\text {th }}$ 
International Conference on Software, Telecommunications and Computer Networks (SoftCOM 2011), pp. 16-21, Sept. 2011.

[2] M.L. Stefanizzi, L. Mottola, L. Mainetti, L. Patrono, "COIN: Opening the internet of things to people's mobile devices", IEEE Communications Magazine, 55 (2), art. no. A1, pp. 20-26, 2017.

[3] FIFA (2015). FIFA Anti-Doping Regulations. Available online at: www.fifa.com

[4] FIFA (2010). F-MARC Nutrition for football. Available online at: www.fifa.com

[5] Ekstrand, J., Hägglund M., Törnqvist H., Kristenson K., Bengtsson H., Magnusson H., Waldén M., "Upper extremity injuries in male elite football players." Knee Surgery, Sports Traumatology, Arthroscopy, vol. 21, no. 7, pp.1626-1632.

[6] M. Maffia, L. Mainetti, L. Patrono, and E. Urso, "Evaluation of potential effects of RFID-based item-level tracing systems on the integrity of biological pharmaceutical products," International Journal of RF Technologies: Research and Applications, vol. 3, no. 2, pp. 101-118, 2012.

[7] D. Alessandrelli, L. Mainetti, L. Patrono, G. Pellerano, M. Petracca, M. L. Stefanizzi, "Performance evaluation of an energyefficient MAC scheduler by using a test bed approach", Journal of Communications Software and Systems, Vol. 9, No. 1, 2013 pp. 84-96.

[8] L. Catarinucci, S. Guglielmi, L. Mainetti, V. Mighali, L. Patrono, M.L. Stefanizzi, L. Tarricone, "An energy-efficient MAC scheduler based on a switched-beam antenna for wireless sensor networks," Journal of Communications Software and Systems, 9 (2), pp. 117-127, 2013

[9] IEEE 802.15.4 Standard. Available Online at: http://www.ieee802.org.

[10] A. Capone, M. Cesana, D. De Donno, and I. Filippini, "Optimal Placement of Multiple Interconnected Gateways in Heterogeneous Wireless Sensor Networks," NETWORKING 2009, Lecture Notes in Computer Science (LNCS), vol. 5550, pp. 442-455, 2009.

[11] D. De Donno, M.L. Stefanizzi, L. Catarinucci, L. Mainetti, L. Patrono, L. Tarricone, "Integrating passive UHF rfid tags with wsn nodes: Challenges and opportunities", Journal of Communications Software and Systems, vol. 10, no. 2, 2014, pp. 99-106.

[12] L. Catarinucci, D. De Donno, L. Mainetti, L. Palano, L. Patrono, M. L. Stefanizzi, L. Tarricone, "An IoT-Aware Architecture for Smart Healthcare Systems", IEEE Internet of Things Journal, 2015, doi:10.1109/JIOT.2015.2417684

[13] C. Bormann, A.P. Castellani, and Z. Shelby, "CoAP: An Application Protocol for Billions of Tiny Internet Nodes", IEEE Internet Computing, Vol. 16, No.2, 2012, pp. 62-67.

[14] S. Tennina, M. Di Renzo, E. Kartsakli, F. Graziosi, A. S. Lalos, A. Antonopoulos, P. V. Mekikis, and L. Alonso, "WSN4QoL: a WSN- oriented healthcare system architecture," International Journal of Distributed Sensor Networks, vol. 2014, 16 pages, 2014.

[15] S.-J. Jung, R. Myllyla, and W.-Y.Chung, "Wireless machine-tomachine healthcare solution using android mobile devices in global networks", IEEE Sensors Journal, vol. 13, no.2, pp. 14191424, May 2013.

[16] P.P. Ray, "Internet of Things for Sports (IoTSport): An architectural framework for sports and recreational activity", 2015 International Conference on Electrical, Electronics, Signals, Communication and Optimization (EESCO), pp. 1-4, Jan. 2015.

[17] M. A. Ikram, M. D. Alshehri, and F. K. Hussain, "Architecture of an IoT-based system for football supervision (IoT Football)",
2015 IEEE 2nd World Forum on Internet of Things (WF-IoT), pp. $69-74$, Dec. 2015.

[18] L. Mainetti, L. Patrono, and M.L. Stefanizzi., "An Internet of Sport Architecture Based on Emerging Enabling Technologies", International Multidisciplinary Conference on Computer and Energy Science, 29 August 2016, Article number 7555928.

[19] D. De Donno, L. Catarinucci, R. Colella, F. Ricciato, and L. Tarricone, "Differential RCS and sensitivity calculation of RFID tags with software-defined radio," 2012 IEEE Radio and Wireless Symposium (RWS 2012), 9-12, Santa Clara, CA, USA, 2012.

[20] Advanticsys, AS-XM1000 resource manual. Available online at: http://www.advanticsys.com/.

[21] Sensor ID, Discovery Gate UHF Datasheet. Available online at: http://www.sensorid.it/

[22] G. K. Teklemariam, J. Hoebeke, I. Moerman, and P. Demeester, "Facilitating the creation of IoT applications through conditional observations in CoAP", EURASIP Journal on Wireless Communications and Networking, June 2013.

[23] Raspberry $\mathrm{Pi}$, User manual. Available online at http://www.raspberrypi.org

[24] A D. Albu, J. Lukkien, and R. Verhoeven. On-node processing of ECG signals. In IEEE CCNC, Jan 2010.

[25] TERTIUM Technology, Blueberry UHF, resource manual. Available online at: http://www.tertiumtechnology.com.

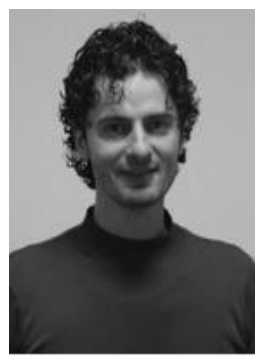

Luca Catarinucci received the Laurea degree (with honors) in Electronic Engineering from the University of Perugia, Italy, in 1998. Since 2003, he has been with the University of Salento, Lecce, Italy, where he is currently an Associate Professor of Electromagnetic Fields and Professor of "Microwaves" and "Electromagnetic Solutions for Hi-Tech" with the Department of Innovation Engineering. He authored more than 150 papers published in international journals and conferences and four chapters books with international diffusion. He holds two patents. His research activity has been mostly focused on the implementation of high-performance electromagnetic simulation tools, on the electromagnetic characterization of heterogeneous materials, on the use of time-domain reflectometry for the qualitative and quantitative characterization of fluids. Currently he is strongly involved in RFIDrelated activities, ranging from antenna and system design, integration between sensors and RFID tags, RFID-based robot navigation, and new techniques for tag characterization, optimization, and design.

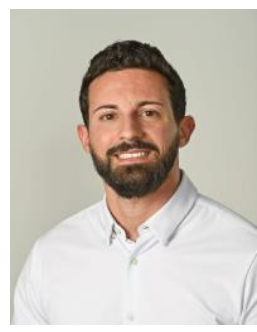

Danilo De Donno received the B.Sc. and M.Sc. degrees (cum laude) in telecommunication engineering from Politecnico di Milano, Italy, in 2005 and 2008, respectively, and the Ph.D. degree in Information Engineering from the University of Salento, Lecce, Italy, in 2012. He was a PostDoctoral Fellow with the ElectroMagnetic Lab Lecce (EML2) of the University of Salento from 2012 to 2015. In July 2015, he joined the Pervasive Wireless Systems Group and the Wireless Networking Group at the IMDEA Networks Institute, Madrid, Spain, as a Post-Doc Researcher. His areas of interest lie in mm-Wave communications and wireless LANs, with main research focus on the development of efficient schemes for beam training and tracking through hybrid analog-digital beamforming. 


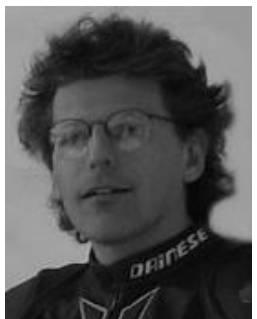

Luca Mainetti is an associate professor of software engineering and computer graphics at the University of Salento. His research interests include web design methodologies, notations and tools, services oriented architectures and IoT applications, and collaborative computer graphics. He is a scientific coordinator of the GSA Lab - Graphics and Software Architectures Lab and IDA Lab - IDentification Automation $\mathrm{Lab}$ at the Department of Innovation Engineering, University of Salento. He is the Rector's delegate at the ICT.

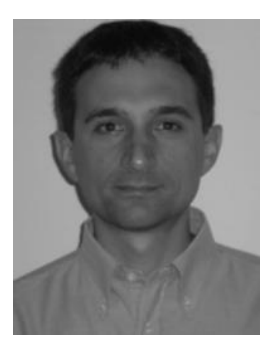

Luigi Patrono received his MS in Computer Engineering from University of Lecce, Lecce, Italy, in 1999 and $\mathrm{PhD}$ in Innovative Materials and Technologies for Satellite Networks from ISUFI-University of Lecce, Lecce, Italy, in 2003. He is an Assistant Professor of Network Design at the University of Salento, Lecce, Italy. His research interests include RFID, Internet of Things, Wireless Sensor Networks, embedded systems, and software architecture for the IoT. He is Chair Organizer of some workshop and symposium focused on IoT topic. He is author of about 100 scientific papers published on international journals and conferences and four chapters of books with international diffusion.

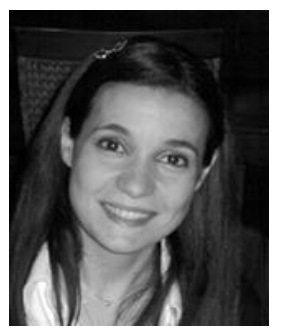

Maria Laura Stefanizzi received her M.Sc. degree (cum laude) in Computer Engineering and her Ph.D. in Information Engineering from University of Salento (Italy), in 2012 and 2016, respectively. She is a Post-Doctoral Fellow with the IDA Lab - IDentification Automation Laboratory at the Department of Innovation Engineering, University of Salento. Her main research activity concerned the integration of the most important Internet of Things (IoT) enabling technologies and their use in order to develop novel Smart Environments and applications.

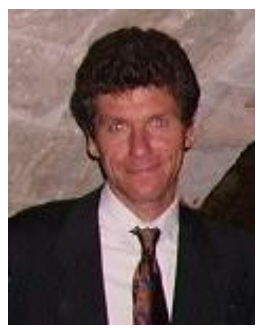

Luciano Tarricone received the Laurea degree (cum laude) in electronic engineering and the Ph.D. degree from Rome University La Sapienza, Rome, Italy, in 1989 and 1994, respectively. Since 1994, he has been a Researcher with the University of Perugia, Italy, and since 1998, he has been a Professore Incaricato of EM fields and EM compatibility. Since 2001, he has been a Faculty Member with the Department of Innovation Engineering, University of Salento, Lecce, Italy, where he is a Full Professor of electromagnetic fields. He has authored over 300 scientific papers. His main contributions are in the modeling of microscopic interactions of EM fields and biosystems, and in numerical methods for efficient computer-aided design (CAD) of microwave circuits and antennas. He is currently involved in bioelectromagnetics, EM energy harvesting and wireless power transmission, novel CAD tools and procedures for MW circuits, RFID, and EM high-performance computing. 